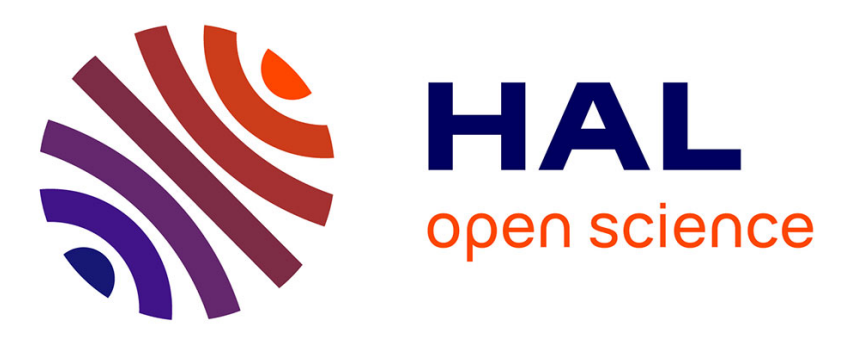

\title{
Modulated SiC nanowires: Molecular dynamics study of their thermal properties
}

Konstantinos Termentzidis, Thibaut Barreteau, Yuxiang Ni, Samy Merabia, Xanthippi Zianni, Yann Chalopin, Patrice Chantrenne, Sebastian Volz

\section{- To cite this version:}

Konstantinos Termentzidis, Thibaut Barreteau, Yuxiang Ni, Samy Merabia, Xanthippi Zianni, et al.. Modulated SiC nanowires: Molecular dynamics study of their thermal properties. Physical Review B: Condensed Matter and Materials Physics (1998-2015), 2013, 87 (12), 125410 / 8p. 10.1103/PhysRevB.87.125410 . hal-01431385

\section{HAL Id: hal-01431385 \\ https://hal.univ-lorraine.fr/hal-01431385}

Submitted on 11 Feb 2021

HAL is a multi-disciplinary open access archive for the deposit and dissemination of scientific research documents, whether they are published or not. The documents may come from teaching and research institutions in France or abroad, or from public or private research centers.
L'archive ouverte pluridisciplinaire HAL, est destinée au dépôt et à la diffusion de documents scientifiques de niveau recherche, publiés ou non, émanant des établissements d'enseignement et de recherche français ou étrangers, des laboratoires publics ou privés. 


\title{
Modulated SiC nanowires: Molecular dynamics study of their thermal properties
}

\author{
Konstantinos Termentzidis, ${ }^{1,2, *}$ Thibaut Barreteau, ${ }^{2}$ Yuxiang Ni, ${ }^{3}$ Samy Merabia ${ }^{4}$ Xanthippi Zianni, ${ }^{5}$ Yann Chalopin, ${ }^{3}$ \\ Patrice Chantrenne, ${ }^{6}$ and Sebastian Volz ${ }^{3}$ \\ ${ }^{1}$ LEMTA-UMR7563, Université de Lorraine and CNRS, 54506 Vandoeuvre Cedex, France \\ ${ }^{2}$ CETHIL-UMR5008, INSA de Lyon and CNRS, 69621 Villeurbanne, France \\ ${ }^{3}$ EM2C UPR CNRS 288 École Centrale Paris, 92295 Châtenay-Malabry, France \\ ${ }^{4}$ LPMCN-UMR5586, Université de Lyon 1 and CNRS, 69621 Villeurbanne, France \\ ${ }^{5}$ Department of Applied Sciences, Technological Education Institution of Chalkida, Psachna, 34400 Evia, Greece \\ ${ }^{6}$ MATEIS-UMR5510, INSA de Lyon and CNRS, 69621 Villeurbanne, France
}

(Received 30 October 2012; revised manuscript received 7 February 2013; published 12 March 2013)

\begin{abstract}
The thermal conductivity of diameter and polytype modulated $\mathrm{SiC}$ nanowires is predicted using nonequilibrium molecular dynamics. For the polytype modulated nanowires, the two main $\mathrm{SiC}$ polytypes, zinc blende $(3 C)$ and wurtzite $(2 \mathrm{H})$ were considered. We show that the thermal conductivity of the diameter modulated nanowires may be even smaller than that of the constant diameter nanowire with the small section. This remarkable reduction in thermal conduction is attributed to a significant thermal boundary resistance displayed by the constriction, as measured by independent molecular-dynamics simulations. The constriction resistance is related to the confinement of low-frequency modes, as shown by vibrational density-of-states calculations. We used Monte Carlo simulations to conclude that the value of the constriction resistance may be explained by the specular reflections of this class of modes on the surface surrounding the constriction.
\end{abstract}

DOI: 10.1103/PhysRevB.87.125410

PACS number(s): 65.40.-b, 07.05.Tp, 68.35.Ja, 44.10.+i

\section{INTRODUCTION}

Advances in nanofabrication technologies have meant that nanostructures and nanostructured materials are easily fabricated, while their applications in molecular electronics, quantum computers, actuators, sensors, and molecular machine are rapidly spreading. ${ }^{1}$ Their novel functions involve unique mechanical, thermal, and electronic properties. A crucial question is whether or not they efficiently transfer heat current and whether they remain mechanically stable at a given operational temperature.

Silicon carbide $(\mathrm{SiC})$ is an important semiconductor with a wide electronic band gap, and has attracted early interest because of its high mechanical strength, high chemical stability, and high thermal conductivity, which make it an interesting candidate for microelectronic applications. It is used in nanoelectronics and its outstanding hardness makes $\mathrm{SiC}$ suitable for devices working in extreme environments. Novel hydrogen storage devices and a series of applications in catalysis, biomedical, and optics are some of the uses for this material. $^{2}$

$\mathrm{SiC}$ sustains a variety of stable nanostructures, like nanowires, nanotubes or nanocages, nanobelts, nanorods, etc. ${ }^{3-6} \mathrm{SiC}$ also exhibits a large number of lattice structure and stacking fault polytypes, making the material an interesting candidate for a great number of applications. ${ }^{1,4,7,8}$ This pronounced polytypism counts more than 200 crystalline modifications. ${ }^{9}$ The synthesis of nanowire superlattices (NWSLs) made of a variety of dissimilar ${ }^{10-13}$ or the superposition of different polytypes of the same material ${ }^{2,4,14}$ have both been suggested in literature in this field. Chemical vapor deposition, arc discharge, carbon nanotubes in vapor-solid or solid-solid reactions have been used to fabricate $\mathrm{SiC}$ nanowires and nanostructured materials. ${ }^{7,15,16}$ It has been shown that such nanostructures exhibit different electronic, optical, and transport properties from those displayed by plain nanowires. ${ }^{17}$
Phonon confinement observed in semiconductor nanowires modifies the phonon velocities, while surface and interfacial scattering increases the thermal resistance. ${ }^{18,19}$ Measuring temperatures and heat fluxes with a nanometer scale resolution is difficult, and such measurements are most often carried out using atomic simulations, which may help in understanding the microscopic phenomena at play. ${ }^{20,21}$ Molecular dynamics (MD) simulations allows the prediction of the thermal transport properties of semiconductors and insulators because in these materials, energy is mainly carried by phonons. Among the MD simulation approaches to calculate the thermal conductivity, there are two main methods: the nonequilibrium (NEMD) method based on forcing a temperature gradient on the system and the equilibrium (EMD) method within the Green-Kubo approach. ${ }^{22-24}$

In this paper, we shall study the homogeneous nanowire superlattices of $\mathrm{SiC}$ with diameter and lattice structure modulation. We will show that the band-structure engineering based on the significant geometrical modulation of the nanowires is responsible for the reduction of thermal conductivity. Surface effects cannot explain this transport behavior and the reduction of the thermal conductivity. ${ }^{25,26}$ The use of other potentials than semiempirical would probably impact the structural relaxation but not the thermal transmission. We shall demonstrate that NWSLs offer also unique thermal properties, which can be promising for high thermoelectric energy conversion. ${ }^{27,28}$ We considered only periodic modulation profiles, but it has been proved that nonperiodic modulation can further decrease the thermal conductivity, which is attributed to the small transmittivity. ${ }^{25,29-31}$ We should mention at this point that the diameter modulated nanowires could be more or less easily fabricated depending mainly on the choice of the material. From the elaboration point of view, structural and geometry modulations are possible even if no $\mathrm{SiC}$ nanowires

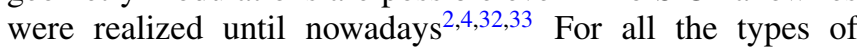
nanowires studied here, we have first relaxed and equilibrated 
the structures using Nosé-Hoover thermostat for sufficient simulation time depending the total number of atoms. Even if we have not proceeded to the relaxation of the nanowires using DFT ab initio calculations, we believe that the diameter modulated nanowires are experimentally feasible, as there is theoretical evidence that such sharp edges are realistic in practice. ${ }^{34}$ Generally speaking, there is an increasing interest in the thermal properties of modulated nanowires the last couple of years, ${ }^{25,26,30,31}$ for both their technological applications and on a theoretical basis in the context of phonon confinement. The two recent papers on the thermal properties of modulated nanowires describe the diffusive phonon transport regime. ${ }^{25,26}$

The outline of the present paper is as follows: in Sec. II below, the thermal conductivity of the two main polytypes of $\mathrm{SiC}$ nanowires as a function of their cross sections and lengths are calculated. These reference values will then be compared to the thermal conductivity of diameter and lattice structure modulated SiC nanowires. In Sec. III, we shall introduce the constriction resistance caused by the necks formed by the small diameter wire section for the diameter modulated nanowires, and also EMD and NEMD predictions are detailed. We will investigate the partial density of states around these constrictions to explain the thermal conductivity decrease of the diameter modulated nanowires compared to that of constant diameter nanowires in Sec. IV. Finally, we shall propose a theoretical analysis of the constriction resistance observed in MD simulations in Sec. V.

\section{THERMAL CONDUCTIVITY OF SIC NANOWIRES WITH CONSTANT AND MODULATED SECTIONS}

The two main polytypes of $\mathrm{SiC}$ are studied in this section, namely the hexagonal $(2 H)$ and the cubic $(3 C)$. These polytypes are characterized by the stacking sequence of the biatomic layers of the $\mathrm{SiC}$ structure in the $c$ direction, which is the $-A B$ - for the $2 H$ and the $-A B C$ - for the $3 C$ polytype. In this section, the thermal conductivity is calculated by the NEMD method only. The NEMD method is similar to the hot-plate experimental setup and in this method a temperature difference is imposed to the edges of the structure. Once we know the heat flux and temperature gradient the thermal conductivity can be calculated using the Fourier law. ${ }^{35-40}$ The LAMMPS code ${ }^{41}$ is used for MD simulations and the interatomic forces are calculated thanks to the Tersoff potential. ${ }^{42,43}$ Free boundary conditions are used in the $x$ and $y$ directions, while in the $z$ direction the use of fixed atoms belonging to the two bilayers at the edges of the nanowire ensures the stability of the nanowires. The mean temperature is set to $300 \mathrm{~K}$, while a temperature difference of $30 \mathrm{~K}$ is imposed between the two thermostats situated at the two edges under the fixed atom layers. We used the temp/rescale command of the LAMMPS code ${ }^{41,44}$ to impose the thermostat temperatures. This command resets the temperature of a group of atoms by explicitly rescaling their velocities. The time step is set to $0.1 \mathrm{fs}$ and the simulation runs depend on the size of the studied nanowires, beginning from $1,000,000$ to $5,000,000$ time steps.

At $300 \mathrm{~K}$ the experimental thermal conductivity of bulk $3 C \mathrm{SiC}$ is of the order of $300 \mathrm{~W} / \mathrm{mK},{ }^{45}$ while the predicted values by means of molecular dynamics are of the order of $400 \mathrm{~W} / \mathrm{mK}^{46,47}$ The purpose of the current paper is not the reproduction of bulk thermal conductivity and instead focuses on the relative changes in thermal conductivity on nanowires due to the modulations.

In Table I, we present a summary of the different systems studied with NEMD. The results of the thermal conductivity are provided in the following subsections.

\section{A. Thermal conductivity of $\mathrm{SiC}$ nanowires with constant cross section}

The thermal conductivity of the $2 \mathrm{H}$ and $3 \mathrm{C}$ polytypes of $\mathrm{SiC}$ nanowires is calculated as a function of their cross sections [Fig. 1(a)] for a constant length equal to $25.5 \mathrm{~nm}$ and as a function of their lengths [Fig. 1(b)] for a constant cross section equal to $15.7 \mathrm{~nm}^{2}$. The thermal conductivity of the $2 H$ polytype is higher than that of the $3 C$ polytype for both studies (cross-section and length dependencies), but as Fig. 8 shows, the extrapolated thermal conductivity for infinite-size nanowires (whose phonon mean free path is much smaller when compared to the nanowires' size) and large cross section favors the $3 C$ nanowires. We also found that the thermal conductivity increases as increasing both the length and the cross section of the nanowires before reaching a plateau. This convergence of thermal conductivity is related to the decoupling of the characteristic dimensions of the nanowires with the phonon mean free path. The enhanced boundary scattering and confinement decrease the thermal conductivity when the system size of the nanowires is decreased. The reduction of the thermal conductivity of the nanowires can reach a factor of 50 compared to that of the bulk material. The same trend was observed for other regular silicon or other semiconductor nanowires. ${ }^{48}$ These results comply well with previous findings published by Papanikolaou ${ }^{19}$ (3.5$4.5 \mathrm{~W} / \mathrm{mK}$ for nanowires with $\mathrm{Si}$ or $\mathrm{C}$ atoms termination). We should stress at this point that all the nanowires studied here have the same kind of termination and studying the effect of the atomic termination of the nanowire was not within the scope of our study.

\section{B. Thermal conductivity of diameter and lattice structure modulated $\mathrm{SiC}$ nanowires}

With the development of new techniques involving varying the temperature and pressure during the growth process

TABLE I. Recapitulative table of the systems studied.

\begin{tabular}{|c|c|c|c|c|c|}
\hline \multirow[b]{2}{*}{ Polytype } & \multicolumn{2}{|c|}{ Constant diameter } & \multicolumn{2}{|c|}{ Diameter modulation } & \multirow{2}{*}{$\begin{array}{l}\text { Lattice modulation } \\
\qquad 2 H / 3 C\end{array}$} \\
\hline & $2 H$ & $3 C$ & $2 H$ & $3 C$ & \\
\hline Cross sections & 15.6 & $\mathrm{~nm}^{2}$ & $15.67 / 35.25 \mathrm{~nm}^{2}$ & $15.67 / 35.25 \mathrm{~nm}^{2}$ & $15.67 \mathrm{~nm}^{2}$ \\
\hline Figures & & & 3(a) & 3(b) & 4 \\
\hline
\end{tabular}




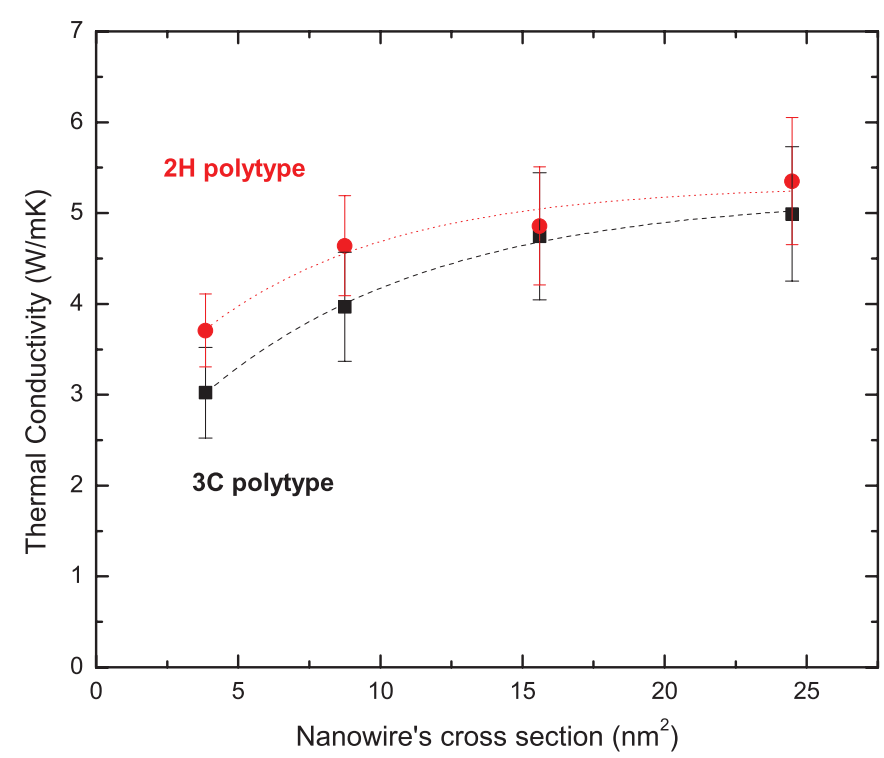

(a)

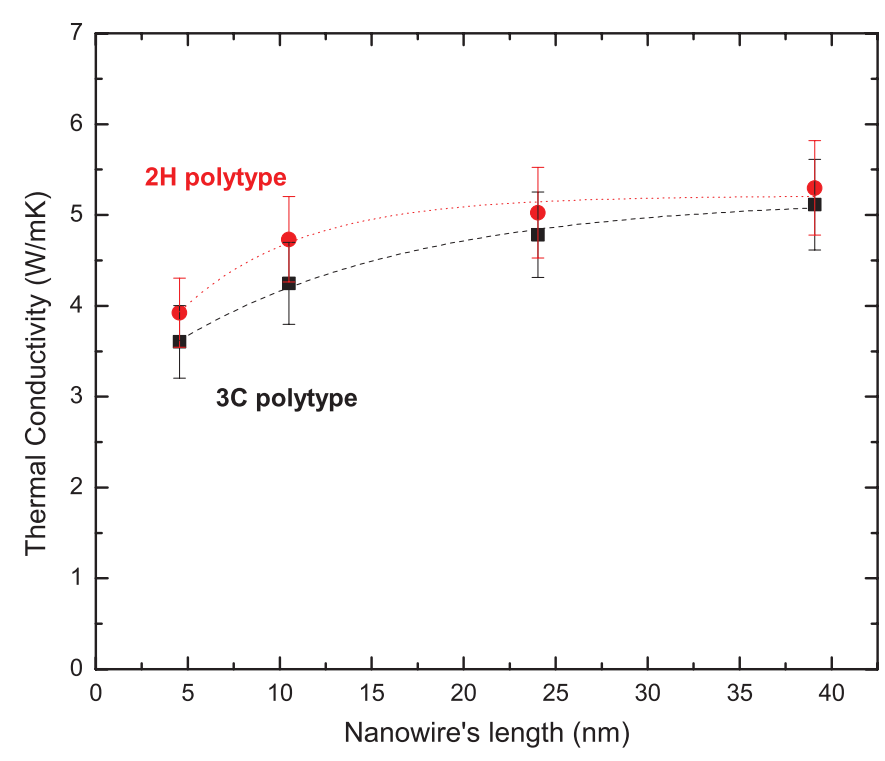

(b)

FIG. 1. (Color online) Thermal conductivity of $2 \mathrm{H}$ and $3 \mathrm{C}$ polytypes of $\mathrm{SiC}$ nanowires as a function of (a) the nanowires' cross section and (b) the nanowires' length.

of nanowires, nanoengineered modulated nanowires have been obtained and reports given in literature in this field. Modulations of the following are possible: the diameter, the lattice structure or the lattice orientation, the doping concentration, and the composition. ${ }^{4,19,27,28,32,33}$ These kinds of modulated nanowires can potentially combine the properties and functions of superlattices and nanowires. The diameter modulated $2 \mathrm{H}$ nanowire considered in our study is depicted in Fig. 2. Results for the thermal conductivity as a function of nanowire length are reported here for the $2 H$ [Fig. 3(a)], and $3 C$ polytype nanowire [Fig. 3(b)]. They are compared to the thermal conductivities of the nanowires with a constant section equal to the smallest and largest sections of the diameter modulation $\left(15.67\right.$ and $\left.35.25 \mathrm{~nm}^{2}\right)$. The calculation of the thermal conductivity for the diameter modulated nanowires was carried out by taking into account the small cross section's pace.

The nanowire's diameter modulation induces heat flux line constriction which increases the thermal resistance of the nanowire compared to a nanowire with a diameter equal to the larger diameter of the modulations. In Fig. 3, the thermal

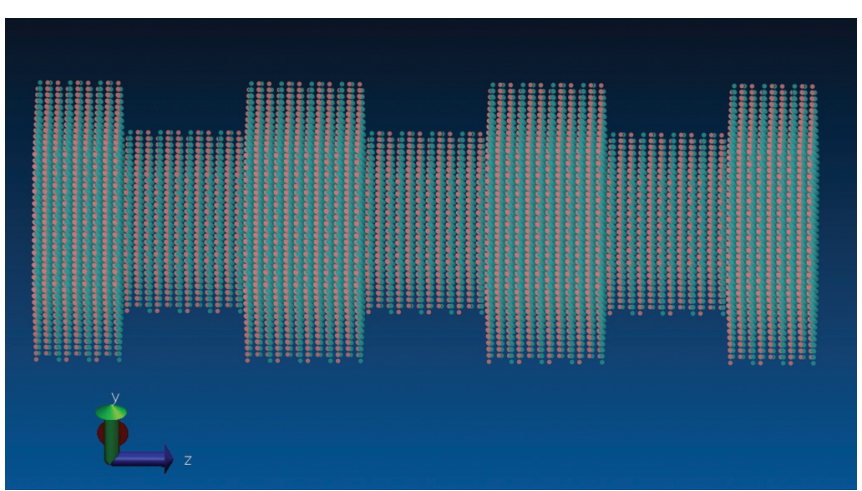

FIG. 2. (Color online) Diameter modulated $2 H$ SiC nanowire. conductivity of the modulated nanowire should thus be smaller than the thermal conductivity of the nanowire with a section of $35.25 \mathrm{~nm}^{2}$ but higher than the thermal conductivity of the nanowire with a section of $15.67 \mathrm{~nm}^{2}$. Actually, the thermal conductivity of the modulated nanowires (both for $2 \mathrm{H}$ and for long enough $3 C$ nanowires) is also smaller than the thermal conductivity of the small cross-section nanowires. Another physical phenomenon, linked with phonon transmission from one section to the other one, might thus be suggested as a reason. This reduction of the thermal conductivity can be attributed to the reduction of the group velocity and the correlation between the wire dimensions and the phonon mean free path, which is around $2 \mathrm{~nm}$ here.

Finally, we also studied the thermal conductivity of the lattice structure modulation. Here the cross section of the nanowire is kept constant and equal to $15.7 \mathrm{~nm}^{2}$. This time, we alternated the two polytypes $2 H$ and $3 C$. Again the modulation affects the thermal conductivity in the same way as the diameter modulation as illustrated by Fig. 4 . The thermal conductivity of the polytype modulated nanowires is smaller than the one of $2 \mathrm{H}$ and $3 \mathrm{C}$, which can be attributed to the existence of a thermal resistance in the polytype modulated nanowires. The thermal resistance and the density of states (DOS) are studied in the next two sections with a view to obtain more in-depth understanding of the physical phenomena related to the reduction of the thermal conductivity of the modulated nanowires.

\section{THERMAL CONSTRICTION RESISTANCE}

We probed the thermal boundary resistance, using both NEMD and the EMD methods. In the following calculations, we consider the diameter modulation of the $2 \mathrm{H} \mathrm{SiC}$ nanowire over just one period (and therefore just one interface) for the sake of computational time. The thermal resistance of a diameter modulated nanowire is the sum of the internal thermal 

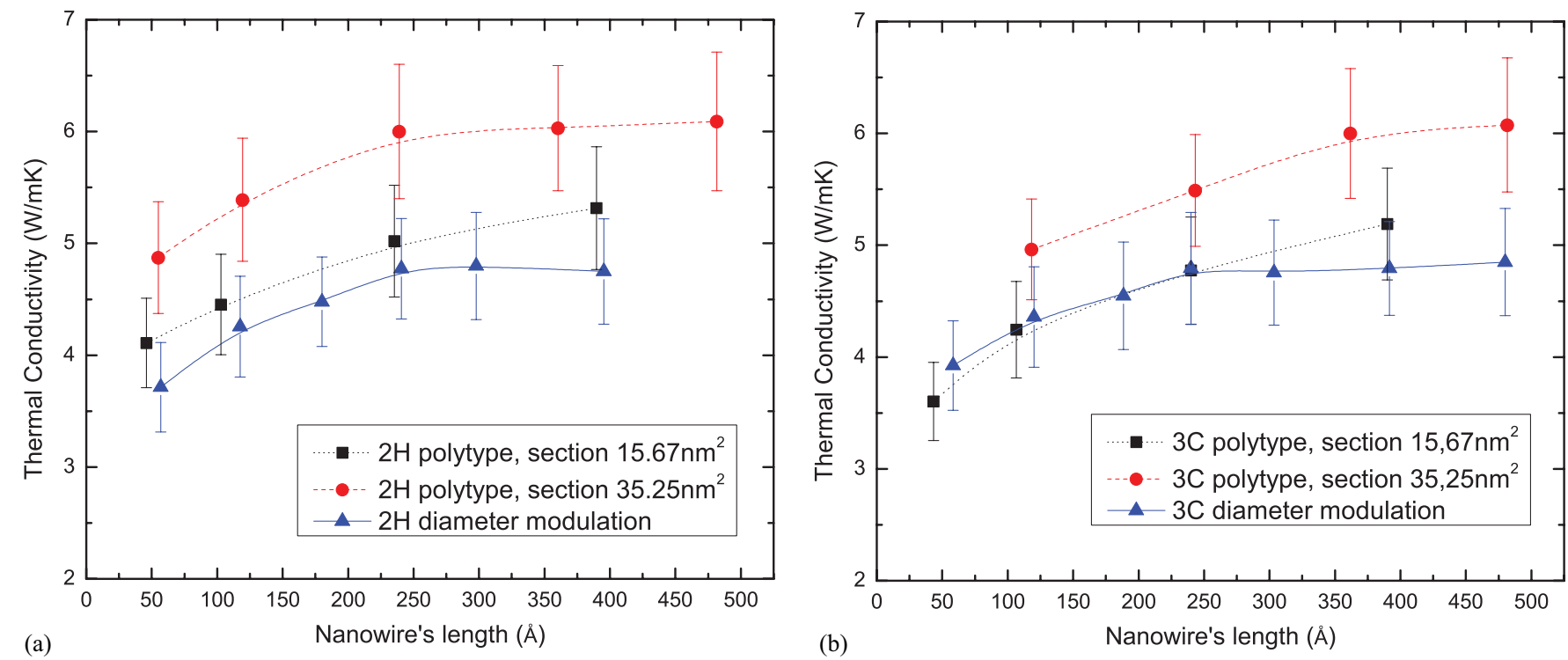

FIG. 3. (Color online) Thermal conductivity of (a) $2 H$ and (b) $3 C$ polytypes of constant diameter SiC nanowires with two different cross sections and the diameter $2 \mathrm{H}$ and $3 \mathrm{C}$ modulated $\mathrm{SiC}$ nanowires as a function of the nanowire's length. The two sections of the diameter modulated nanowires are equal to 15.67 and $35.25 \mathrm{~nm}^{2}$.

resistance of each constant cross section of the nanowire and the constriction resistance near the interface. The constriction resistance differs from the Kapitza resistance, which is the thermal resistance at the interface between two different solids.

In the EMD method the thermal boundary resistance $R$ between two systems with temperature difference $\Delta T$ could be calculated using the following equation: ${ }^{49}$

$$
R_{T R} k_{B}=\int_{0}^{\infty} \frac{\langle\Delta T(0) \Delta T(t)\rangle}{\left\langle\Delta T(0)^{2}\right\rangle} d t\left(\frac{1}{N_{1}}+\frac{1}{N_{2}}\right),
$$

where $N_{1}$ and $N_{2}$ refer to the number of degrees of freedom of the two parts in thermal contact, respectively. By calculating

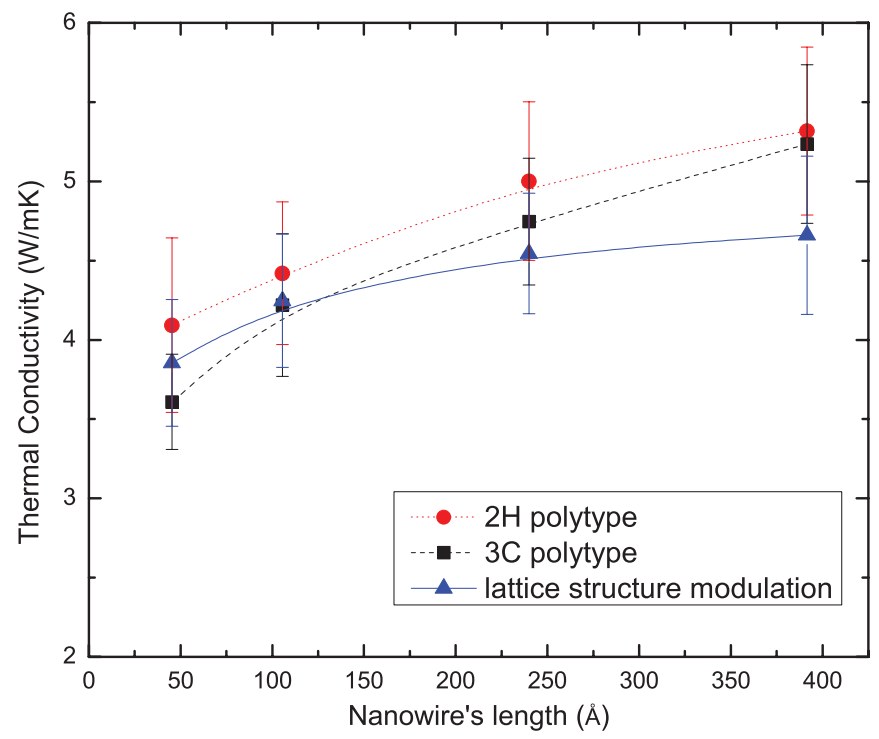

FIG. 4. (Color online) Thermal conductivity of constant diameter $2 \mathrm{H}$ and $3 \mathrm{C}$ polytypes and the lattice structure modulated $\mathrm{SiC}$ nanowires $(2 \mathrm{H} / 3 \mathrm{C})$. The section of the nanowires is equal to $15.67 \mathrm{~nm}^{2}$ the autocorrelation function $(\mathrm{ACF})$ of the temperature difference fluctuations at equilibrium, the thermal resistance could be obtained. While the temperatures could be acquired in MD from the kinetic energies, the final ACFs were derived from the average of twenty trajectories with different initial velocities. The constriction resistance for diameter modulated nanowires is calculated with the EMD method and found to be equal to $2.37 \times 10^{-10} \mathrm{~m}^{2} \mathrm{~K} / \mathrm{W}$, while the internal thermal resistance for the small and large cross sections are equal to 1.21 and $1.30 \times 10^{-10} \mathrm{~m}^{2} \mathrm{~K} / \mathrm{W}$, respectively.

The NEMD method is based on the calculation of temperature difference at the two sides of the interfaces and the knowledge of the heat flux $q$ in such a way that $R=\Delta T / q$. With the NEMD method, the average internal thermal resistance for $2 H \mathrm{SiC}$ nanowires is equal to $0.6 \times$ $10^{-10} \mathrm{~m}^{2} \mathrm{~K} / \mathrm{W}$. The diameter modulated nanowires show a constriction resistance of $0.9 \times 10^{-10} \mathrm{~m}^{2} \mathrm{~K} / \mathrm{W}$. Possible explanations for the discrepancies between the EMD and NEMD values of the thermal resistances are discussed in a recent paper of Merabia. ${ }^{22}$

Using the analogy between the thermal and the electrical resistance, the total thermal resistance equivalent to one period would be

$$
\begin{aligned}
R_{\mathrm{Tot}} & =R_{S W}+2 R_{T R}+R_{L W} \\
& =\frac{L_{S W}}{\lambda_{S W} S_{S W}}+2 R_{T R}+\frac{L_{L W}}{\lambda_{L W} S_{L W}} \\
& =7.44 \times 10^{-9} \mathrm{~m}^{2} \mathrm{~K} / \mathrm{W},
\end{aligned}
$$

where $R_{i W}(i=S$ or $L)$ is the internal thermal resistance, $L_{i W}$, $S_{i W}, \lambda_{i W}$ are the length, cross section, and thermal conductivity of the small or large cross-section nanowires measured with the NEMD method. Using this total resistance, we calculated the equivalent thermal conductivity with the Fourier law:

$$
\lambda_{\mathrm{Tot}}^{\text {equivalent }}=\frac{L_{\mathrm{Tot}}}{R_{\mathrm{Tot}} S_{S W}}=4.03 \mathrm{~W} / \mathrm{mK},
$$


which complies well with the molecular dynamics thermal conductivity of $4.13 \mathrm{~W} / \mathrm{mK}$ reported in Fig. 3. From this simple model, we can conclude that the reduction of the thermal conductivity of the diameter modulated nanowires is due to the constriction thermal resistance.

The variation of the cross sections also plays a role in the constriction resistance. Using NEMD, we studied two kinds of diameter variations from 8.81 to $15.67 \mathrm{~nm}^{2}$ and from 8.81 to $47.98 \mathrm{~nm}^{2}$. In the first case the constriction resistance value was $0.8 \times 10^{-10} \mathrm{~m}^{2} \mathrm{~K} / \mathrm{W}$ and $1.2 \times 10^{-10}$ $\mathrm{m}^{2} \mathrm{~K} / \mathrm{W}$ in the second. The periodicity of the superlattices does not influence the interfacial resistance. The two methods provide the same trends even if they do not give the same results. The constriction resistance is approximately twice as large as the internal resistance of the nanowire. Finally, the temperature profiles indicates that this resistance is localized in very few monolayers around the nanowire interface between the diameters of the large and the small sections.

\section{DENSITY OF STATES}

The density of states for the case of the diameter modulated $2 \mathrm{H}$ nanowire is studied with cross sections varying from 8.81 to $15.67 \mathrm{~nm}^{2}$. The phonon density of states of the particle is numerically obtained by decomposing the time correlation function of the atomic velocities into the Fourier space a ${ }^{50,51}$

$$
P(\omega)=\frac{1}{k_{B} T} \sum_{i} m_{i}\left|v_{i}(\omega)\right|^{2},
$$

where $\omega$ denotes the angular frequency.

In Fig. 5, the partial density of states is calculated for the small and large cross sections far from the interfaces and for the first adjacent layers on both sides of the interfaces (Fig. 6). We focused on three areas: (a) For intermediate frequencies between 25 and $30 \mathrm{THz}$ where there is a decrease in the

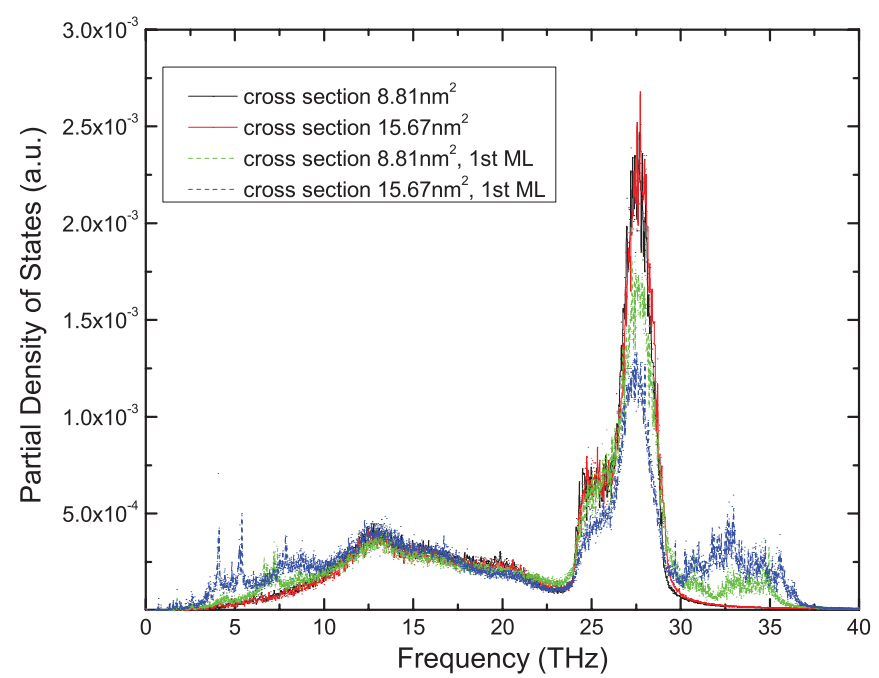

FIG. 5. (Color online) Density of states for four distinct groups (see Fig. 6). The black and red lines correspond to the groups of atoms far from the surface for small $\left(8.81 \mathrm{~nm}^{2}\right)$ and large $\left(15.67 \mathrm{~nm}^{2}\right)$ cross sections. The green line refers to the DOS in the small section and the first layer, which is the closest to the interface, while the blue one is the DOS in the large section and in the first layer, which is closest to the interface.

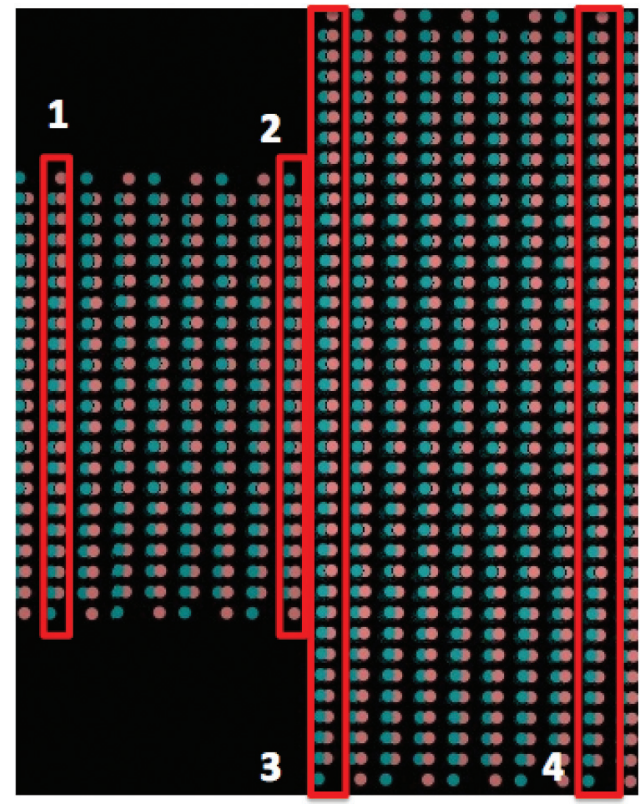

FIG. 6. (Color online) The four considered groups for the calculation of the partial density of states. 1: Group of atoms far from the interface for the small cross section nanowire $\left(8.81 \mathrm{~nm}^{2}\right)$; 2: group of atoms for the first bilayer closest to the interface; 3,4 : groups of atoms for the large $\left(15.57 \mathrm{~nm}^{2}\right)$ cross-section nanowire far from and the first bilayer closest to the interface.

density of states of the adjacent layers in comparison to ones of the constant section nanowires; note that this main peak is generated by the $\mathrm{SiC}$ bond; (b) for small frequencies between 0 and $10 \mathrm{THz}$ [Fig. 7(a)] where confined modes appear in the first layer of the large cross section and as peaks related to the stationary phonons; (c) for frequencies ranging from 30 to $37 \mathrm{THz}$ where the additional local maxima of the density is attributed to the interfacial and surface phonon modes [Fig. 7(b)].

The DOS at the first layer in the large cross section exhibits additional low-frequency peaks [see Fig. 7(b) for more details]. These low-frequency peaks are only shown at the cross section layers, indicating phonon confinement. Similar phenomena are also observed in glasses. ${ }^{52-54}$ We identified these frequencies as being very close to frequencies of the blocked transverse and longitudinal phonons. These phonons cannot pass from the small cross section to the larger or vice versa, as they correspond to unavailable frequencies. The phonon interactions might be altered due to this confinement and as a result a higher Kapitza resistance was found for diameter modulated nanowires.

\section{THEORETICAL ANALYSIS}

Generally speaking, the interfacial resistance may be written as the sum of three contributions: ${ }^{.5}$

$$
R=R_{\text {Maxwell }}+R_{\mathrm{b}}+R_{\text {diffr }},
$$

where $R_{\text {Maxwell }}$ is the Maxwell resistance due to the heat flux lines' constriction in the vicinity of the orifice, $R_{\mathrm{b}}$ is the resistance due to phonons having a mean free path larger than the orifice radius $\alpha$ and which are transported ballistically. Finally, $R_{\text {diffr }}$ is the resistance due to the diffraction of the 

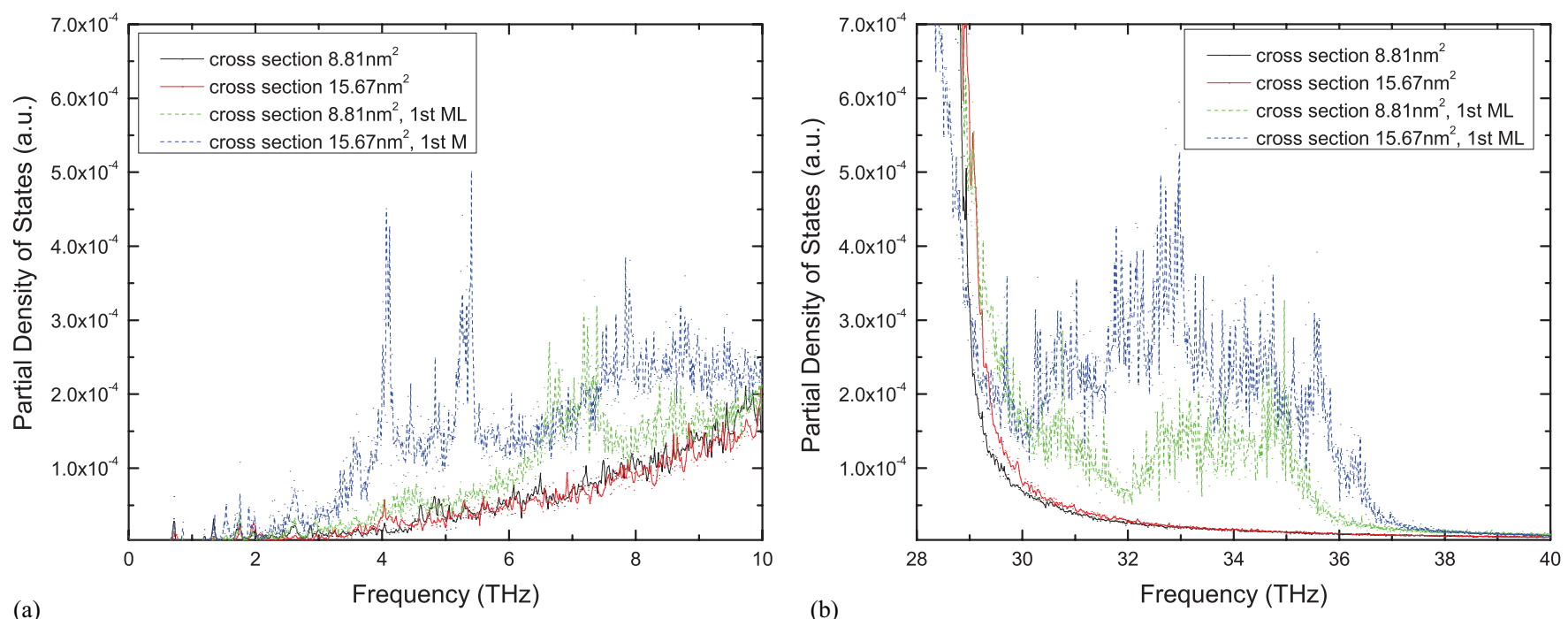

FIG. 7. (Color online) Density of states for four distinct groups (see Fig. 6) for frequencies (a) 0-10 THz and (b) 28-40 THz.

phonons having a wavelength comparable or smaller than $\alpha$. The latter resistance is negligible because we were working at a temperature where the phonon correlation length is very small. The first two contributions have a simple general expression and depend on the thermal conductivity of the material:

$$
R_{\text {Maxwell }}=\frac{\pi \alpha}{2 \lambda}
$$

and

$$
R_{\mathrm{b}}=\frac{4}{c_{v} v},
$$

where $c_{v}$ is the volumetric specific heat and $v$ is the average acoustic velocity of the material. For $\mathrm{SiC}$, one can estimate $R_{\mathrm{b}} \simeq 2.4 \times 10^{-10} \mathrm{~m}^{2} \mathrm{~K} / \mathrm{W}$ in the classical regime relevant to MD where $c_{v}=\rho k_{B}, \rho$ being the number density. This ballistic resistance is the one measured in any section of a nanowire without constriction and should correspond to the value obtained in EMD. In the presence of a constriction, the resistance should increase because a fraction of the incoming phonons is not transmitted by the constriction. Different effects may explain this phonon blocking: first, because of the difference in cross sections, some modes allowed to propagate in the large cross section nanowire may be blocked by the constriction and prevented from transmitting. To quantify this effect, we may write the components of a propagating wave vector as

$$
\vec{k}=2 \pi\left(\frac{n_{x}^{p}}{D_{p}} \vec{e}_{x}+\frac{n_{y}^{p}}{D_{p}} \vec{e}_{y}+\frac{n_{z}^{p}}{h} \vec{e}_{z}\right)
$$

in the nanowire of large cross section which is here supposed to be cylindrical with a diameter $D_{p}$ and a length $h, \vec{z}$ denoting the axis of the nanowire. The indexes $n_{x}^{p}, n_{y}^{p}$, and $n_{z}^{p}$ are integers which take values between 1 and $D_{p} / a$ where $a$ is the bulk $\mathrm{SiC}$ lattice parameter. The propagating modes in the small cylinder may be parametrized in the same way. If we assume that a given mode $\vec{k}$ conserves its momentum at the passage of the constriction, certain low wave vector modes will be confined within the large cylinder. These correspond to values of the indexes $n_{x}^{p}$ and $n_{y}^{p}$ spanning the interval $\left[1 ; D_{p} / D_{m}\right]$ where $D_{m}$ is the diameter of the small cylinder.
The contribution of these confined modes to the constriction resistance may be estimated by using the expression of the flux:

$$
\begin{aligned}
q= & \frac{1}{V_{p}} \sum_{\vec{k} \text { allowed }} k_{B} b v\left(T_{p}-T_{m}\right)=\frac{1}{V_{p}} \sum_{\text {all } \vec{k}} k_{B} b v\left(T_{p}-T_{m}\right) \\
& -\frac{1}{V_{p}} \sum_{\text {blocked } ; \vec{k}} k_{B} b v\left(T_{p}-T_{m}\right),
\end{aligned}
$$

where $V_{p}=h \pi\left(D_{p} / 2\right)^{2}$ is the volume of the big cylinder. For the sake of simplicity, we assumed a dispersionless medium characterized by an average group velocity $v$ and a number $b$ of polarization branches. $T_{p}$ and $T_{m}$ denote the temperatures of the large and small cylinders which are supposed to be constant. The first term on the right-hand side of Eq. (9) is simply $q=\left(T_{p}-T_{m}\right) / R_{b}$ where $R_{b}$ is the ballistic resistance. The second term may be recast and yields a correction to the ballistic resistance:

$$
\delta R \simeq R_{b}^{2} \frac{\pi\left(D_{p} / 2\right)^{2} a}{k_{B} b v\left(D_{p} / D_{m}-1\right)^{2}} .
$$

For the constriction between the two cylinders with cross sections $S_{p}=35.25 \mathrm{~nm}^{2}$ and $S_{m}=15.67 \mathrm{~nm}^{2}$, this yields an additional resistance $\delta R \simeq 2.8 \times 10^{-13} \mathrm{~m}^{2} \mathrm{~K} / \mathrm{W}$ which is thus small compared to the ballistic resistance. We conclude that the value of the constriction resistance cannot be explained solely by wave physics. Another contribution to the constriction resistance is purely geometric. Some phonons traveling in the large section may be reflected by the annular disk surrounding the constriction. Because the surfaces of the nanowire considered in MD are atomically sharp, these reflections are certainly specular and may greatly enhance the constriction resistance. To quantify this effect, we performed simple Monte Carlo simulations based on the ray-tracing method. ${ }^{56}$ We considered a constriction between two cylindrical cylinders with cross sections $S_{p}=35.25 \mathrm{~nm}^{2}$ and $S_{m}=15.67 \mathrm{~nm}^{2}$, respectively, and a length $h=5 \mathrm{~nm}$. The boundaries of the two cylinders are connected to two heat reservoirs at the fixed temperatures $T_{p}$ and $T_{m}$. Each reservoir is supposed to play the role of a black body emitting phonons in 


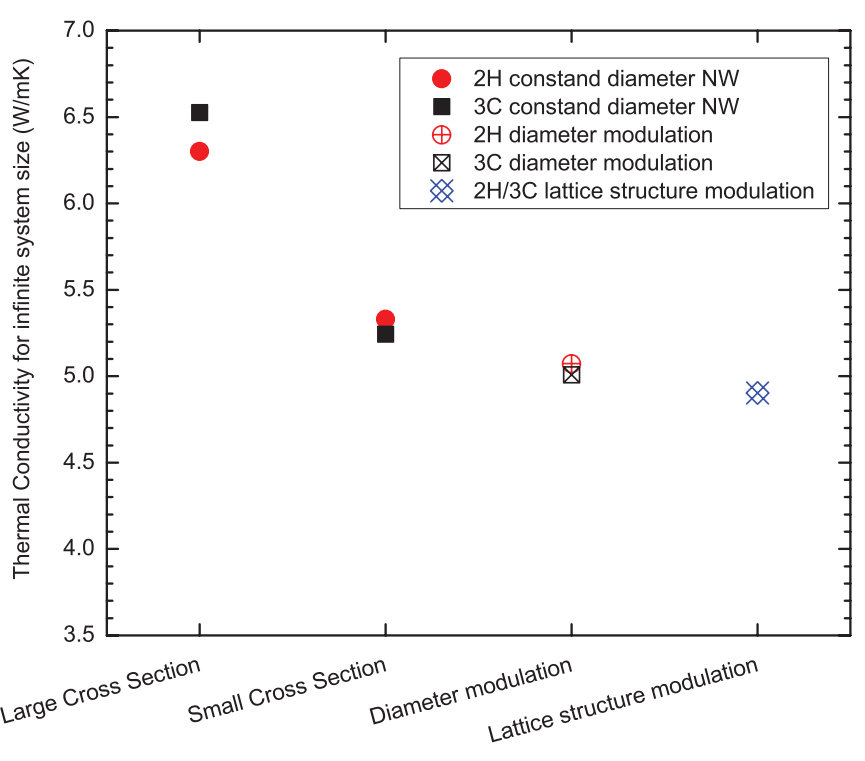

FIG. 8. (Color online) Thermal conductivity for infinite system size for the constant cross section $2 H$ and $3 C$ monotype NWs, the diameter modulated $2 \mathrm{H}$ and $3 \mathrm{C}$ nanowires, and the lattice modulated $2 \mathrm{H} / 3 \mathrm{C}$ nanowires.

the direction $\theta$ of the constriction. We used a spatial resolution of $0.1 \mathrm{~nm}$ and discretized the cosine of the polar angle $\theta$ and the azimuthal angle $\Phi$ using 20 discrete values. For simplicity's sake, we work with a gray assumption and consider a single phonon mean free path $\Lambda=70 \mathrm{~nm}$ characterizing ballistic heat transport in bulk $\mathrm{SiC}$ which sets the probability that a phonon disappears at each time step $d P=v d t / \Lambda \ll 1$. Phonons are then allowed to travel towards the constriction eventually being specularly reflected by the boundaries.

The propagation of a given phonon mode is stopped whenever it reaches one of the two heat reservoirs. It should be noted that because of the eventuality of backscattering, some phonons may reach the reservoir they came from. The heat flux density $q$ flowing across the constriction is estimated using the sum of the heat fluxes arriving at each reservoir divided by the section of the reservoir. When the two heat baths are at the same temperature, we measure a very small net flux compared to $\rho k_{B} v T_{p}=4 T_{p} / R_{b}$. If we impose a difference of temperatures between the reservoirs, we measure a constriction resistance of the order of $2.2 R_{b} \simeq 5.45 \times$ $10^{-10} \mathrm{~m}^{2} \mathrm{~K} / \mathrm{W}$. Approximately, $60 \%$ of the phonons emitted by the heat bath connected to the large nanowire are reflected by the annular disk surrounding the constriction. Among these phonons, $80 \%$ are backscattered to the large heat bath from which they were emitted. It should also be noted that on average, each phonon experiences 2.8 reflections on the surface of the large cylinder (not including the annular disk) and 1.8 reflection events on the surface of the small cylinder. The value of the constriction resistance obtained is larger than the EMD value. However, the Monte Carlo simulations have shown that the presence of the constriction increases the thermal resistance by a factor 2 as compared to the internal resistance of the nanowire, which complies with the molecular dynamics simulation results. This tends to confirm that specular reflections of large phonon mean free paths modes on the surface of the annular disk surrounding the constriction may be at the origin of the resistance enhancement.

\section{CONCLUSIONS}

In the present paper, we predicted the thermal conductivity of the two main polytypes of $\mathrm{SiC}$ nanowires, the $3 C$ and the $2 H$, using molecular dynamics. This calculation for these two types using the dimensions described herein acts as a complement to existing literature. ${ }^{3,8,19}$ The length and the cross section both influence the thermal conductivity for both cases in the same way: increasing the length results in the thermal conductivity increasing until the length becomes larger than the phonon mean free path. Increasing the cross section results in an increase to thermal conductivity.

The study of the diameter and the lattice structure modulated nanowires show the thermal conductivity of a given nanowire with those two modulations can be reduced further (Fig. 8). This is due to the reduction of the phonon transport generated by the additional free surfaces and the additional thermal interfacial resistance.

We have defined this thermal resistance as a constriction resistance and have shown that it is worth twice the internal thermal resistance of the constant diameter nanowire. The density of states study shows that phonon modification is related to the confinement of the modes and additional surface modes appear with the diameter modulation. Monte Carlo simulations have also shown that this increase in the resistance may be explained by specular reflections on the annular disk surrounding the constriction.

We believe that a certain number of phonons are trapped in the large cross sections and this might be a helpful way of achieving the objective of further reducing the thermal conductivity of nanowires for application with thermoelectric materials, for example. A combined modulation of diameter and lattice structure might even reduce further the thermal conductivity of nanowires. It will be interesting to study the phonon transmission coefficient at these constrictions. We believe that the transmission coefficient will provide a clearer picture of the phenomenon and may help give more detailed information on the physical mechanisms at play in the constriction resistance.

\footnotetext{
*konstantinos.termentzidis@univ-lorraine.fr

${ }^{1}$ D. Srivastava, M. A. Makeev, M. Menon, and M. Osman, J. Nanosci. Nanotechnol. 8, 3628 (2008).

${ }^{2}$ R. Wu, B. Li, M. Gao, J. Chen, Q. Zhu, and Y. Pan, Nanotechnology 19, 335602 (2008).

${ }^{3}$ J. W. Lyver and E. Blaisten-Barojas, J. Comput. Theor. Nanosci. 8, 529 (2011).
}

${ }^{4}$ D.-H. Wang, D. Xu, Q. Wang, Y.-J. Hao, G.-Q. Jin, X.-Y. Guo, and K. N. Tu, Nanotechnology 19, 215602 (2008).

${ }^{5}$ A. K. Ray and M. N. Huda, J. Comput. Theor. Nanosci. 3, 315 (2006).

${ }^{6}$ W. Zhou, X. Liu, and Y. Zhang, Appl. Phys. Lett. 89, 223124 (2006). ${ }^{7}$ G. Shen, D. Chen, K. Tang, Y. Qian, and Y. Zhang, Chem. Phys. Lett. 375, 1772003. 
${ }^{8}$ P. Chantrenne and K. Termentzidis, Phys. Status Solidi A 209, 2492 (2012).

${ }^{9}$ A. Zywietz, K. Karch, and F. Bechstedt, Phys. Rev. B 54, 1791 (1996).

${ }^{10}$ M. Gudiksen, L. Lauhon, J. Wang, D. Smith, and C. Lieber, Nature (London) 415, 617 (2002).

${ }^{11}$ Y. Wu, R. Fan, and P. Yang, Nano Lett. 2, 83 (2002).

${ }^{12}$ R. Solanki, J. Huo, J. L. Freeouf, and B. Miner, Appl. Phys. Lett. 81, 3864 (2002).

${ }^{13}$ A. Zhang, L. C. Lew Yan Voon, and M. Willatzen, Phys. Rev. B 73, 045316 (2006).

${ }^{14}$ H. J. Choi, H.-K. Seong, J.-C. Lee, and Y.-M. Sung, J. Cryst. Growth 269, 472 (2004).

${ }^{15}$ D. Zhang, A. Alkhateeb, H. Han, H. Mahmood, D. N. McIlroy, and M. Grant Norton, Nano Lett. 3, 983 (2003).

${ }^{16}$ Y. Zhang, T. Ichihashi, E. Landree, F. Nihey, and S. Iijima, Science 285, 1719 (1999).

${ }^{17}$ S. Mizuno and N. Nishiguchi, J. Phys.: Condens. Matter 21, 195303 (2009).

${ }^{18}$ C. Dames and G. Chen, J. Appl. Phys. 95, 682 (2004).

${ }^{19}$ N. Papanikolaou, J. Phys.: Condens. Matter 20, 135201 (2008).

${ }^{20} \mathrm{~S}$. Volz and D. Lemonnier, Phys. Low-Dimens. Struct. 5-6, 91 (2000).

${ }^{21}$ S. Volz, D. Lemonnier, and J.-B. Saulnier, Microscale Thermophys. Eng. 5, 191 (2001).

${ }^{22}$ S. Merabia and K. Termentzidis, Phys. Rev. B 86, 094303 (2012).

${ }^{23} \mathrm{~K}$. Termentzidis and S. Merabia, Molecular Dynamics - Theoretical Developments and Applications in Nanotechnology and Energy, edited by Lichang Wang (InTech, 2012), Chap. 5.

${ }^{24}$ J. L. Barrat and F. Chiaruttini, Mol. Phys. 101, 1605 (2003).

${ }^{25}$ X. Zianni and P. Chantrenne, J. Electron. Mater. (2012).

${ }^{26}$ D. L. Nika, A. I. Cocemasov, C. I. Isacova, A. A. Balandin, V. M. Fomin, and O. G. Schmidt, Phys. Rev. B 85, 205439 (2012).

${ }^{27}$ X. Zianni, Appl. Phys. Lett. 97, 233106 (2010).

${ }^{28}$ X. Zianni, Nanoscale Res. Lett. 6, 1 (2011).

${ }^{29}$ M. K. Y. Chan, J. Reed, D. Donadio, T. Mueller, Y. S. Meng, G. Galli, and G. Ceder, Phys. Rev. B 81, 174303 (2010).

${ }^{30}$ Y. He and G. Galli, Phys. Rev. Lett. 108, 215901 (2012).

${ }^{31}$ X. Zianni, J. Solid State Chem. 193, 53 (2012).

${ }^{32}$ P. Caroff, K. A. Dick, J. Johansson, M. E. Messing, K. Deppert, and L. Samuelson, Nat. Nanotechnol. 4, 50 (2009).
${ }^{33}$ R. Algra, M. Verheijen, M. Borgstrom, L. F. Feiner, G. Immink, W. van Enckevort, E. Vlieg, and E. Bakkers, Nature (London) 456 , 369 (2008)

${ }^{34}$ L. Ansari, G. Fagas, J.-P. Colinge, and J. Greer, Nano Lett. 12, 2222 (2012).

${ }^{35}$ K. Termentzidis, J. Parasuraman, C. Abs Da Cruz, S. Merabia, D. Angelescu, F. Marty, T. Bourouina, X. Kleber, P. Chantrenne, and P. Basset, Nanoscale Res. Lett. 6, 1 (2011).

${ }^{36}$ P. K. Schelling, S. R. Phillpot, and P. Keblinski, Phys. Rev. B 65, 144306 (2002).

${ }^{37}$ K. Termentzidis, P. Chantrenne, and P. Keblinski, Phys. Rev. B 79, 214307 (2009).

${ }^{38}$ K. Termentzidis, S. Merabia, P. Chantrenne, and P. Keblinski, Int. J. Heat Mass Transf. 54, 2014 (2011).

${ }^{39} \mathrm{P}$. Chantrenne and J. L. Barrat, Eurotherm 75 "Microscale Heat Transfer 2", Superlattices Microstruct. 35, 173 (2004).

${ }^{40}$ P. Chantrenne and J. L. Barrat, J. Heat Transfer 126, 577 (2004).

${ }^{41}$ S. Plimpton, J. Comput. Phys. 117, 1 (1995).

${ }^{42}$ J. Tersoff, Phys. Rev. Lett. 56, 632 (1986).

${ }^{43}$ J. Tersoff, Phys. Rev. B 39, 5566 (1989).

${ }^{44}$ S. J. Plimpton, R. Pollock, and M. Stevens, in Proceedings of the Eighth SIAM Conference on Parallel Processing for Scientific Computing (SIAM, Minneapolis, Minnesota, 1997).

${ }^{45}$ R. E. Taylor, H. Groot, and J. Ferrier, Thermophysical Properties of CVD SiC (Purdue University, West Lafayette, IN, 1993).

${ }^{46}$ J. Li, L. Porter, and S. Yip, J. Nucl. Mater. 255, 139 (1998).

${ }^{47}$ G. D. Samolyuk, S. I. Golubov, Y. N. Osetsky, and R. E. Stoller, J. Nucl. Mater. 418, 174 (2011).

${ }^{48}$ C. Abs da Cruz, K. Termentzidis, P. Chantrenne, and X. Kleber, J. Appl. Phys. 110, 034309 (2011).

${ }^{49}$ A. Rajabpour and V. Volz, J. Appl. Phys. 108, 094324 (2010).

${ }^{50}$ G. Domingues, J.-B. Saulnier, and S. Volz, Eurotherm 75 "Microscale Heat Transfer 2", Superlattices Microstruct. 35, 227 (2004).

${ }^{51}$ A. Rajabpour, S. M. Vaez Allaei, and F. Kowsary, Appl. Phys. Lett. 99, 051917 (2011).

${ }^{52}$ P. K. Jha, Indian J. Pure Appl. Phys. 44, 87 (2006).

${ }^{53}$ R. Zorn, Phys. Rev. B 81, 054208 (2010).

${ }^{54}$ T. S. Jain and J. J. de Pablo, J. Chem. Phys. 120, 9371 (2004).

${ }^{55}$ R. Prasher, T. Tong, and A. Majumdar, Appl. Phys. Lett. 91, 143119 (2007).

${ }^{56}$ G. Chen, J. Heat Transf. 121, 945 (1999). 